\title{
Far-infrared imager and polarimeter for the Origins Space Telescope
}

\author{
Johannes Staguhn $\odot,{ }^{a, b}, *$ Edward Amatucci $\odot,{ }^{\text {b }}$ Damon Bradley, \\ David Chuss $\odot{ }^{\text {c }}$ James Corsetti, ${ }^{\text {b }}$ Mike DiPirro, ${ }^{\text {b }}$ Dale Fixsen,, b,d \\ Joseph Howard, ${ }^{b}$ David Leisawitz $\odot,{ }^{b}$ S. Harvey Moseley, ${ }^{b}$ \\ Margaret Meixner, ${ }^{\mathrm{e}}$ Alexandra Pope $\odot$, Joaquin Vieira, \\ Edward Wollack $\odot,{ }^{b}$ and the Origins Space Telescope Mission \\ Concept Study Team \\ ${ }^{\mathrm{a} J o h n s ~ H o p k i n s ~ U n i v e r s i t y, ~ N A S A / G o d d a r d ~ S p a c e ~ F l i g h t ~ C e n t e r, ~ D e p a r t m e n t ~ o f ~ P h y s i c s ~}$ \\ and Astronomy, Greenbelt, Maryland, United States \\ ${ }^{b}$ NASA Goddard Space Flight Center, Greenbelt, Maryland, United States \\ ${ }^{\mathrm{c}}$ Villa Nova University, Villanova, Pennsylvania, United States \\ ${ }^{\mathrm{d}}$ University of Maryland, College Park, Department of Astronomy, Maryland, United States

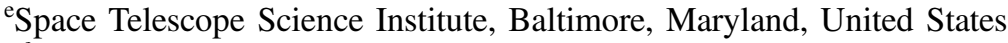 \\ ${ }^{\mathrm{f}}$ University of Massachusetts, Amherst, Massachusetts, United States \\ ${ }^{\mathrm{g}}$ University of Illinois, Urbana, Illinois, United States
}

\begin{abstract}
The far-infrared imager and polarimeter (FIP) for the Origins Space Telescope (Origins) is a basic far-infrared imager and polarimeter. The camera will deliver continuum images and polarization measurements at 50 and $250 \mu \mathrm{m}$. Currently available detector technologies provide sufficient sensitivity for background limited observations from space, at least on a single pixel basis. FIP incorporates large next-generation superconducting detector arrays and our technology development plan will push the pixel numbers for the arrays to the required size of 8000. Two superconducting detector technologies are currently candidates for the instrument: transition edge sensors or microwave kinetic inductance devices. Using these detectors and taking advantage of the cryogenic telescope that is provided by Origins, FIP will achieve mapping speeds of up to eight orders of magnitude faster than what has been achieved by existing observatories. The science drivers for FIP include observations of solar system objects, dust properties, and magnetic field studies of the nearby interstellar medium, and large scale galaxy surveys to better constrain the star formation history of the universe to address one of the main themes of Origins: "How does the Universe work?" In addition to the science, the FIP instrument plays a critical functional role in aligning the mirrors during on orbit observatory commissioning. () The Authors. Published by SPIE under a Creative Commons Attribution 4.0 Unported License. Distribution or reproduction of this work in whole or in part requires full attribution of the original publication, including its DOI. [DOI: 10.1117/1.JATIS.7.1.011016]
\end{abstract}

Keywords: astronomy; detectors; infrared.

Paper 20080SS received Jun. 16, 2020; accepted for publication Feb. 26, 2021; published online Mar. 23, 2021.

\section{Introduction}

The far-infrared (FIR) foreground level in space is very low, ${ }^{1}$ leaving significant room for future space-based missions to achieve more than three orders of magnitude improvement in sensitivity over former missions, such as AKARI ${ }^{2}$ Infrared Space Observatory, ${ }^{3}$ Spitzer, ${ }^{4}$ and Herschel. ${ }^{5}$ This foreground is dominated by zodiacal and galactic emission. Only at wavelengths of $>300 \mu \mathrm{m}$ does the cosmic microwave background radiation begin to contribute significantly as a background source. To be sky-noise limited, space-based FIR telescopes need to be cooled to cryogenic temperatures of $\sim 4 \mathrm{~K}$. Furthermore, low noise detectors with a noise equivalent power (NEP) of $3 \times 10^{-19} \mathrm{~W} / \sqrt{ } \mathrm{Hz}$ for space-based continuum observations are needed to be

*Address all correspondence to Johannes Staguhn, Jstaguhn@jhu.edu 
photon noise limited. ${ }^{1}$ The Origins Space Telescope (Origins) consists of a 5.9-m on-axis telescope cooled to 4.5 degrees Kelvin. This feeds an instrument suite consisting of a mid-infrared spectrometer, specialized for the characterization of transiting exoplanets around M-stars [MidInfrared Spectrometer (Camera) Transit Spectrometer ${ }^{6}$, the far-infrared survey spectrometer OSS, ${ }^{7}$ and the Far-IR imager/polarimeter (FIP), described here. FIP provides imaging and polarimetric measurement capabilities at 50 and $250 \mu \mathrm{m}$. FIP's large surveys take advantage of Origins's ${ }^{8}$ agility: The observatory can scan-map the sky very fast at $60^{\prime \prime}$ per second, which is essential for efficient mapping, as the FIP $250-\mu \mathrm{m}$ channel reaches the extragalactic source confusion limit in a few milliseconds. The fast-mapping mode also enables rapid follow-up of transient or variable sources and enables efficient monitoring campaigns. FIP will enable wide area ( $\left.\geq 1000 \mathrm{deg}^{2}\right)$ photometric surveys, leading to large statistical multiwavelength studies of populations of astronomical objects, complementing ground-based and space-based surveys obtained, e.g., with the Vera Rubin Observatory (VRO) Legacy Survey of Space and Time $\left(\right.$ LSST) ${ }^{9}$ and the Nancy Grace Roman Space Telescope, a 2.5-m optical survey telescope (formerly WFIRST). ${ }^{10}$ A combination of deep and wide unbiased spectroscopic surveys obtained with the onboard FIR spectrometer OSS with wide-area FIP continuum surveys will enable Origins to measure star formation and AGN growth, the rise of metals, and feedback in galaxies over cosmic time and across the cosmic web. ${ }^{11}$

\subsection{Scientific Motivation}

A two-tier continuum survey with FIP covering (1) $500 \mathrm{deg}^{2}$ at 50 and $250 \mu \mathrm{m}$ ("wide"), and (2) $10,000 \mathrm{deg}^{2}$ at $250 \mu \mathrm{m}$ ("ultra-wide") will constrain the role of environment in driving dustobscured star formation and black hole growth, discover large populations of dust-obscured galaxies into the epoch of reionization, and complement planned optical (e.g., VRO/LSST and Eucli d) and space-based near-infrared (e.g., NGRST) surveys by providing a robust measurement of the obscured star formation rates (SFRs).

The FIP wide survey at $50 \mu \mathrm{m}$ can detect more than $99 \%$ of the galaxies detected by the Nancy Roman Space telescope-HLS and provide SFRs and dust masses to complement the stellar mass measurements from the near-infrared. The 50 and $250 \mu \mathrm{m}$ measurements will enable studies of SFRs in galaxies as a function of stellar mass and redshift, which is crucial for our understanding of galaxy evolution (e.g., Ref. 12). The FIP ultra-wide survey will take a census of rare galaxy populations, such as massive and intensely star-forming galaxies (e.g., Ref. 13), QSOs at $z>6$ during the EOR, ${ }^{14}$ strong-gravitationally lensed galaxies, ${ }^{15}$ and protoclusters (Fig. 1). ${ }^{16}$ These exciting new populations of galaxies that will be uncovered by FIP can

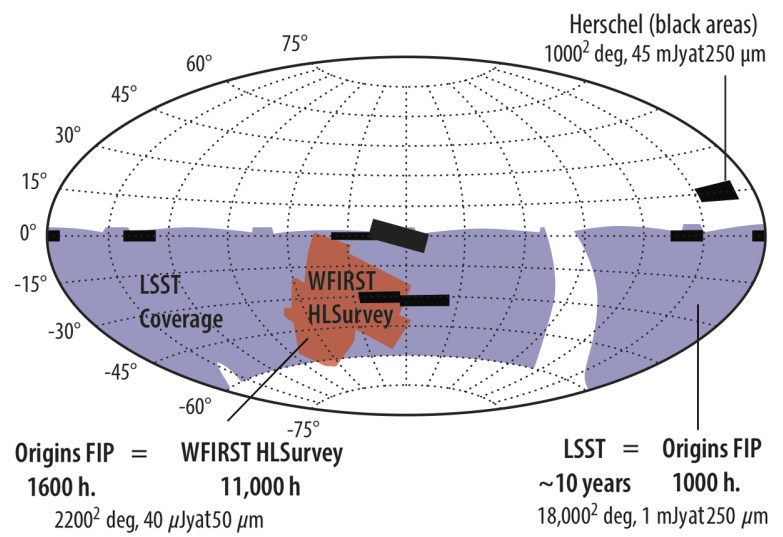

Fig. 1 Origins/FIP can rapidly produce continuum maps over a very large area. This sky projection shows the footprint of the two widest surveys, LSST and WFIRST-HLS, and the time estimate to cover those areas with FIP. The proposed FIP surveys would include a wide, $10,000 \mathrm{deg}^{2}$ at $250 \mu \mathrm{m}$ and a medium, $500 \mathrm{deg}^{2}$ area at 50 and $250 \mu \mathrm{m}$ that would provide SFRs and dust masses to complement LSST and WFIRST-HLS stellar masses and detect $>99 \%$ of the galaxies that will be seen with the Nancy Roman Space telescope in the near infrared. 
be followed up by OSS for detailed characterization. For more details on FIP's science case see Ref. 11.

Additionally, with FIP's large field of view (FOV), which can be used to obtain beam maps on point sources with large dynamic range, the instrument will be ideally suited to align the mirrors during on-orbit observatory commissioning using phase retrieval holography. ${ }^{17}$

\section{Enabling Technology}

FIP's major enabling technology is provided by the availability of superconducting detectors, which, on a single pixel basis, have been demonstrated to provide a noise performance that will enable background limited observations with Origins. There are currently two potential technologies considered for the detectors: transition edge sensors (TES) or microwave kinetic inductance devices (KIDs), see also Ref. 18. Examples of existing arrays using these technologies are shown in Fig. 2. TES are currently the baseline detector technology for FIP. FIP's mapping speed will be enabled by arrays with a pixel count of 8000 . The efficient readout of the detectors can be achieved by cryogenic microwave superconducting quantum-interference devices ( $\mu$ Wave SQUID) multiplexers that have been developed at National Institute of Standards Boulder. ${ }^{19,20}$ Those multiplexers allow for a multiplexing factor of 2000, which results in only $4 \mathrm{RF}$ readout lines for the array.

Sub-Kelvin coolers are the other main enabling technology for FIP. The FIP detectors, like the OSS detectors, are cooled to $0.05 \mathrm{~K}$ by a continuous adiabatic demagnetization refrigerator $\left(\mathrm{CADR}^{21}\right)$. The path to maturation of these technologies is described in the Origins' Technology Development Plan, a supplement to the full report. ${ }^{22}$

\section{Operational Principles}

The FIP functional block diagram (Fig. 3) shows the optical, cryogenic, and electronics components in the FIP design. The path of the incoming radiation to the detector is shown in pink. FIP has a pick-off-mirror, fore-optics, half-wave plates (HWP) on mechanisms, a barrel mechanism with two sets of optics, leading through a filter to the detector plane. The CADR assembly controls the required $\mathrm{mK}$ temperatures for the detectors. Launch locks, mechanical structure, and pre-amplifier electronics and harnessing complete the design.

In pointed or survey imaging and polarimetry mode, FIP observes in one of the two bands, either 50 or $250 \mu \mathrm{m}$. A single detector with a $109 \times 73$ pixel format ( 2000 pixels) is used for all measurements. The wavelength is selected by the optics/filter combination that can be switched with the barrel mechanism. In polarization mode, a HWP modulator and an analyzer (polarizing grid) is brought into the beam. In this setup, the relevant stokes parameters $I, Q$, and $U$ are being measured. These measurements fully characterize the linearly polarized emission from aligned dust grains, enabling the targeted polarimetry science goals.

FIP provides diffraction-limited images with an angular resolution of $[1 / 23 \times$ wavelength $(\mu \mathrm{m})](\operatorname{arcsec})$ and covers an instantaneous FOV of $3.6^{\prime} \times 2.5^{\prime}$ at $50 \mu \mathrm{m}$ and $13.5^{\prime} \times 9^{\prime}$ at
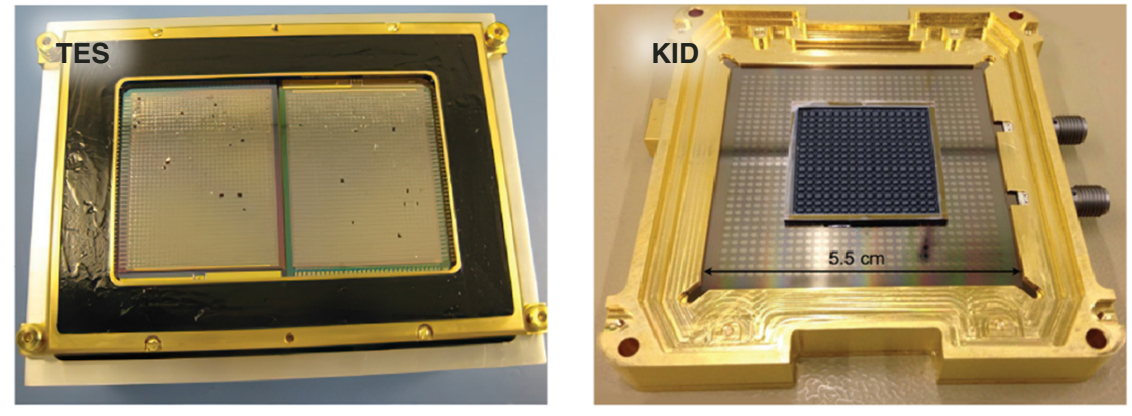

Fig. 2 The Origins Technology Development Plan recommends parallel investment in two detector technologies for FIP: TES bolometers and KIDS. 


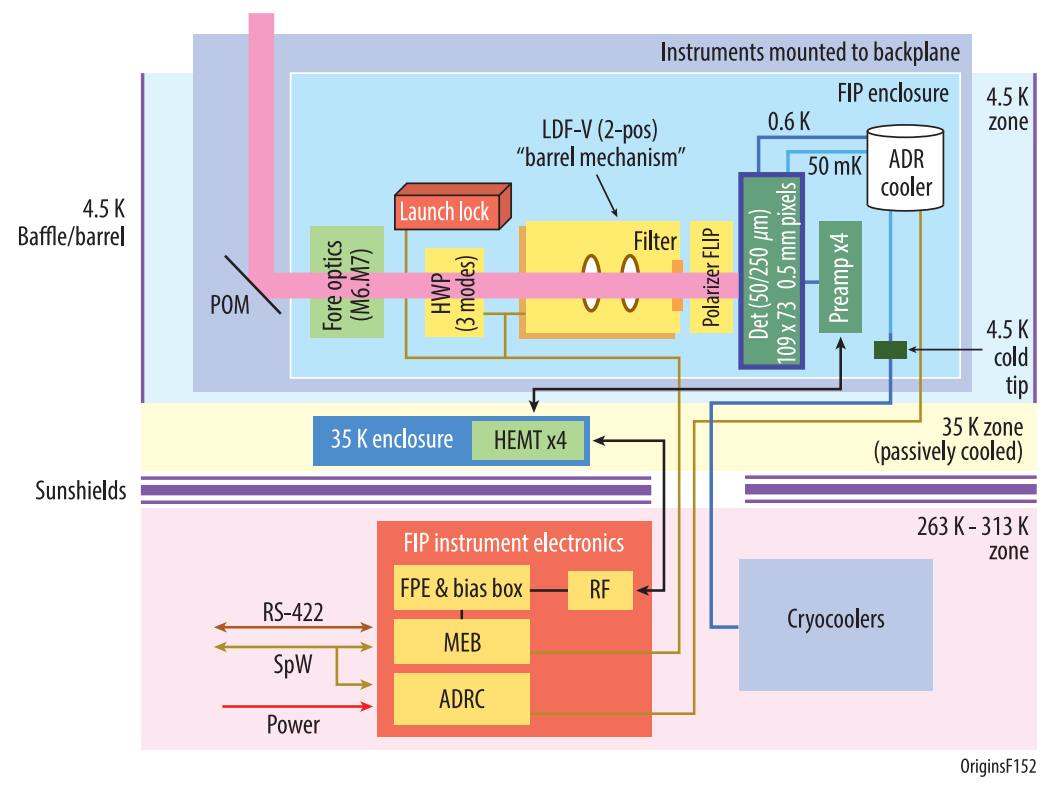

Fig. 3 FIP has a simple design with one detector selectable by filter to 50 or $250 \mu \mathrm{m}$. This functional block diagram of FIP shows how the power dissipation in the cold $4.5 \mathrm{~K}$ section is minimized by placing the readout electronics in the warm part of the observatory.

$250 \mu \mathrm{m}$. FIP has an optimized design to meet the maximum achievable optical efficiency in each band while requiring only a moderate FOV from the telescope. Either the field steering mirror (FSM) for pointed observations, or the telescope for survey mapping moves at all times during FIP observations to link the detector response of all pixels within the $1 / f$ time interval (i.e., at a frequency higher than that of the $1 / f$ noise knee, typically $<0.1 \mathrm{~Hz}$ for TES). For single pointing observations, only the FSM moves. Several observing patterns can be chosen, for example, a Lissajous Pattern for small maps, or a chessboard pattern for large surveys. ${ }^{23}$ For survey mapping, the observatory sweeps the telescope pointing in raster patterns to map significant areas of the sky, up to hundreds or more square degrees. This operational principle has the significant benefit that the telescope does not need to settle at a certain position on the sky. For reconstruction of an image, only the knowledge of where the telescope has been pointed at any moment in time is needed. For Origins, the star trackers, combined with the gyroscopes deliver this knowledge.

\section{Optical Design}

The FIP optical system design allows for a compact configuration (Figs. 4 and 5). Light is directed into the FIP instrument box by a pickoff mirror located near the telescope focal surface.

Inside the instrument box, a Dragone ${ }^{24}$ collimator comprising a pair of free-form (polynomial surface) mirrors (CM1 and CM2), is used to collimate the light and correct aberrations (Fig. 6). While free-form mirrors would be more difficult to construct than traditional conic shaped ones at the same operating wavelength, we do not think they would be a technical challenge to manufacture given that the long wavelength affords much looser surface figure and roughness tolerances relative to optical surfaces.

Depending on the mode of operation, the collimated beam either traverses or bypasses a HWP before passing through an open window or a polarizer. For each of the two bands, the rays are imaged onto the detector array by a dedicated lens system, which provides the same beam-sampling factor for each, 50 and $250 \mu \mathrm{m}$. Like the HWP and polarizer, the lens system switches in and out during operation depending on the waveband. The system uses an $f / 2$ or $f / 8$ lens for the short and long bands, respectively. The HWP, polarizer, and lenses are all switched in and out through mechanisms that are described in Sec. 8. 


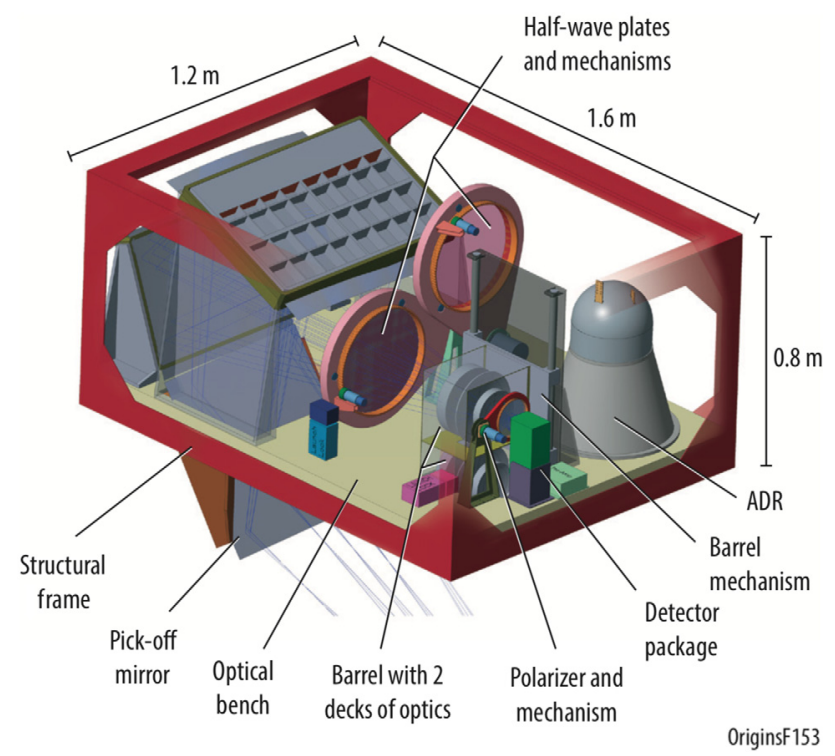

Fig. 4 The FIP mechanical structure and components. The labels point out individual components described in the text.

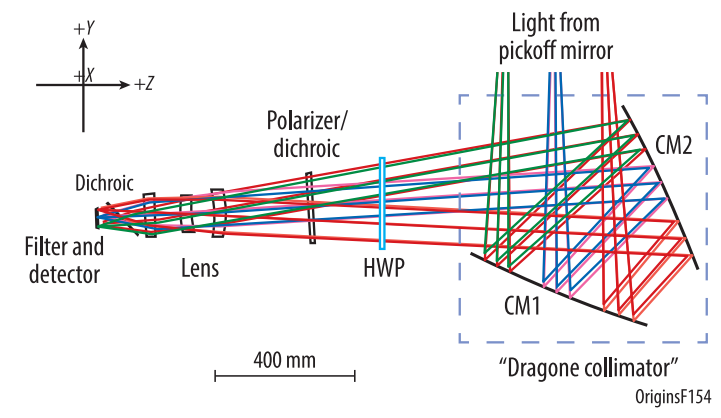

Fig. 5 The FIP optical system minimizes the number of optical components. This configuration shows the layout for a polarization measurement because the HWP is in the beam.

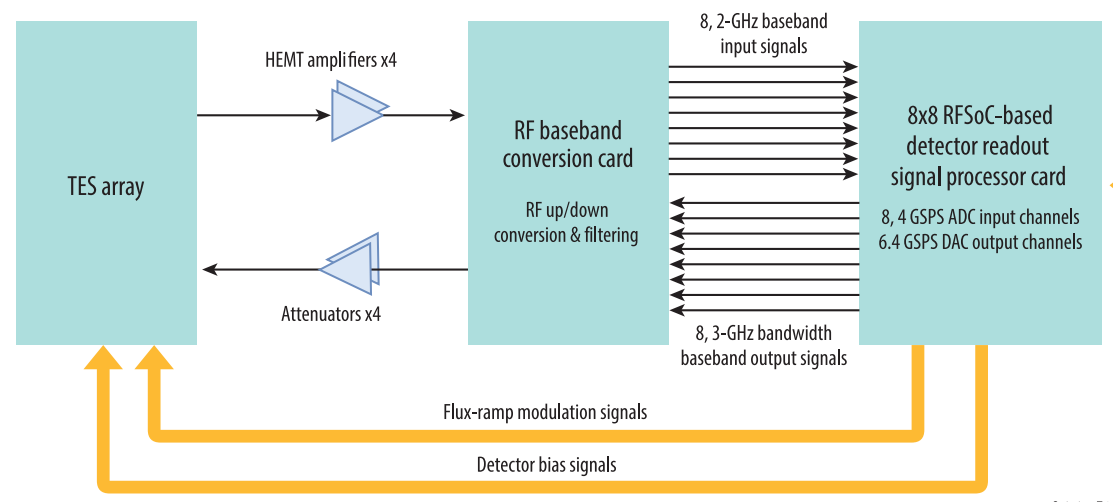

OriginsF155

Fig. 6 Detector readout scheme for FIP: FIP and RFSoC samples and processes signals simultaneously

\section{Observing Modes}

FIP observing modes (Table 1) include continuum and polarimetry observations. Either type of observation can be done in pointed or survey mapping modes. Pointed observations are 
Table 1 Instrument parameters.

\begin{tabular}{|c|c|c|c|c|c|c|}
\hline $\begin{array}{l}\text { Observing } \\
\text { mode }\end{array}$ & $\begin{array}{l}\text { Wavelength } \\
\text { coverage } \\
(\mu \mathrm{m})\end{array}$ & $\begin{array}{l}\text { Angular } \\
\text { resolution }\end{array}$ & FOV & $\begin{array}{c}\text { Spectral } \\
\text { resolving power } \\
(P=\lambda / \Delta \lambda)\end{array}$ & $\begin{array}{l}\text { Saturation } \\
\text { limits }\end{array}$ & $\begin{array}{c}\text { Representative } \\
\text { sensitivity } \\
5 \sigma \text { in } 1 \mathrm{~h}\end{array}$ \\
\hline \multirow[t]{2}{*}{ Pointed } & \multirow[t]{2}{*}{$\begin{array}{c}50 \text { or } 250 \\
\text { (selectable) }\end{array}$} & $50 / 250 \mu \mathrm{m}$ & $50 \mu \mathrm{m}: 3.5^{\prime} \times 2.5^{\prime}$ & \multirow[t]{2}{*}{3.3} & $\begin{array}{l}50 \mu \mathrm{m}: \\
1 \mathrm{Jy}\end{array}$ & $\begin{array}{l}50 / 250 \mu \mathrm{m:} \\
120 \mathrm{nJy} / 1.1 \mathrm{mJy}\end{array}$ \\
\hline & & $2.5^{\prime \prime} / 12.5^{\prime \prime}$ & $\begin{array}{c}250 \mu \mathrm{m}: 13.5^{\prime} \times 9^{\prime} \\
(109 \times 73 \text { pixels })\end{array}$ & & $\begin{array}{l}250 \mu \mathrm{m}: \\
5 \mathrm{Jy}\end{array}$ & $\begin{array}{l}\text { Confusion limit: } \\
50 / 250 \mu \mathrm{m}: \\
0.9 / 2.5 \mu \mathrm{Jy}\end{array}$ \\
\hline \multirow[t]{2}{*}{$\begin{array}{l}\text { Survey } \\
\text { mapping }\end{array}$} & \multirow[t]{2}{*}{$\begin{array}{l}50 \text { or } 250 \\
\text { (selectable) }\end{array}$} & $50 / 250 \mu \mathrm{m}$ & $\begin{array}{c}\text { Above FOV } \\
\text { moving at } 60^{\prime \prime} / \mathrm{s}\end{array}$ & 3.3 & $\begin{array}{c}50 \mu \mathrm{m}: \\
1 \mathrm{Jy}\end{array}$ & \multirow{2}{*}{$\begin{array}{l}\text { Same as above, } \\
\text { time to reach } \\
\text { confusion limit: } \\
50 \mu \mathrm{m}: 1.9 \mathrm{~h} \text {; } \\
250 \mu \mathrm{m}: 2 \mathrm{~ms}\end{array}$} \\
\hline & & $2.5^{\prime \prime} / 12.5^{\prime \prime}$ & & & $\begin{array}{l}250 \mu \mathrm{m}: \\
5 \mathrm{Jy}\end{array}$ & \\
\hline \multirow{2}{*}{$\begin{array}{l}\text { Polarimetry } \\
\text { pointed or } \\
\text { mapping }\end{array}$} & \multirow[t]{2}{*}{$\begin{array}{c}50 \text { or } 250 \\
\text { (selectable) }\end{array}$} & $50 / 250 \mu \mathrm{m}$ & $50 \mu \mathrm{m}: 3.5^{\prime} \times 2.5^{\prime}$ & 3.3 & $\begin{array}{l}50 \mu \mathrm{m}: \\
2 \mathrm{Jy}\end{array}$ & \multirow{2}{*}{$\begin{array}{l}0.1 \% \text { in linear } \\
\text { polarization } \\
\pm 1 \text { deg in pol. } \\
\text { angle }\end{array}$} \\
\hline & & $2.5^{\prime \prime} / 12.5^{\prime \prime}$ & $\begin{array}{c}250 \mu \mathrm{m}: 13.5^{\prime} \times 9^{\prime} \\
(109 \times 73 \text { pixels })\end{array}$ & & $\begin{array}{l}250 \mu \mathrm{m}: \\
10 \mathrm{Jy}\end{array}$ & \\
\hline
\end{tabular}

performed when the observatory points at the source target, whereas the FSM performs a small Lissajous scan that moves the target sources on the central part of the detector array to provide the required pixel to pixel crosslinks. The second observing mode is survey mapping: the observatory moves in a raster form over the sky, whereas the FSM can be used to create more crosslinks between pixels in one $1 / f$ time, if the raster scan cannot accommodate this criterion.

\section{Detector Subsystems}

The FIP requirements could be met with multiple detector approaches, ${ }^{16}$ and all of them require some development. Our path to create FIP detectors is described in detail in the Origins Technology Development Plan. ${ }^{22}$ However, for our studied design, we have adopted superconducting TES bolometers as the baseline.

The TES are membrane-suspended and close-packed with the individual pixels directly coupling to the observed photons. The imaging array for FIP consists of $\sim 8000$ TES elements (pixels), configured in a rectangular shape $(109 \times 73)$. The TES detectors are densely multiplexed by $\mu$ Wave SQUIDs multiplexers: ${ }^{18,19}$ About 2000 channels can be read out over the $4 \mathrm{GHz}$ readout bandwidth of the $\mu$ Wave SQUID. Cryogenic high-electron-mobility transistor (HEMT) amplifiers are used in combination with room temperature readout electronics to facilitate readout and signal processing.

Each TES has its own microwave resonator (built into the $\mu$ Wave SQUID). Since roughly 2000 resonators fit within a $4 \mathrm{GHz}$ processing bandwidth, and 8000 pixels need to be read out, a total of four radio frequency (RF) signals need to be connected to corresponding HEMT amplifiers and transmitted downstream for signal processing.

\section{Readout Electronics}

The FIP RF signals will be sampled and processed using the commercially available Xilinx Radio Frequency System-on-Chip (RFSoC), which is not yet space certified. The detector feedback and other current signals needed for the detector readout can also be controlled by the RFSoC in combination with digital electronics on the same board (Fig. 6).

\subsection{FIP Data Rates}

The data rate is determined by the pixel count (8000) and the sampling rate is determined by the speed at which the detector projection on the sky is moving, which establishes the required

J. Astron. Telesc. Instrum. Syst. 011016-6 Jan-Mar 2021 • Vol. 7(1) 
detector time constant of a few milli-seconds. The dynamic range of the detector together with the readout speed determines the required bit depth. The FIP detectors will have a time constant of about $1 \mathrm{~ms}$. Since the TES integrates the signal for the duration of its time constant, a "typical" visit time of $3 \mu \mathrm{s}$ per pixel every $\sim 10^{-4} \mathrm{~s}$ is sufficient. The sensitivity requirement for photon noise dominated operation of the TES is $3.10^{-19} \mathrm{~W} / \mathrm{sqrt}(\mathrm{Hz}){ }^{22}$ Thus the power level traced by the lowest significant bit (LSB) will be $\sim 3 \mathrm{e}-21 \mathrm{~J}$. Typically we will have $\sim 100$ samples so each sample LSB only needs to correspond to the $3 \mathrm{e}-20 \mathrm{~J}$ level. At the other end we need to be able to handle $200 \mathrm{fW}$ for $110^{-4} \mathrm{~s}$, or $2 \mathrm{e}-17 \mathrm{~J}$. This power level corresponds to the saturation limit of the TES. ${ }^{22}$ So at the sample rate we are baselining, we will have to accommodate a dynamic range of $2 e-17 / 3 e-20=667$ or 9.4 bits. Hence 12 bits are more than sufficient.

In practice we would not be at saturation and looking for minimum noise simultaneously. We are looking at some level and using the 12 bits to look at changes from that level. For example, if the background is close to $100 \mathrm{fW}$, we can use the 12 bits to look at variations around that level and we would normally have more than 2.6 bits margin. For the transmission of the data, we assume we can co-add detector data down to a rate of $100 \mathrm{~Hz}$. This increases the potential dynamic range by a factor of $\sim 10$. Using 16 bits would be sufficient, but if we dynamically select the bits for the transmission, and even if there are occasional peaks that are beyond 12 bits, we can still bit-compress the full data set to well under 12 bits/pixel per uploaded sample.

The otherwise uncompressed data rate for 8000 pixels thus will be equal to or $<10 \mathrm{Mbits} / \mathrm{s}$. Depending on the observed sources, the lossless compression rate should be between two and four (the latter for a deep survey on the sky with many weak sources), bringing the transmission rate down to between 2.5 and $5 \mathrm{Mbits} / \mathrm{s}$.

\section{Thermal and Mechanical Design}

\subsection{Thermal Architecture}

The FIP thermal system shares design features with the OSS instrument thermal design described in Ref. 7. Both instruments benefit from significant overlap and synergy in technology maturation and design methods. The FIP optics and mechanisms operate at $4.5 \mathrm{~K}$ with cooling provided by mechanical cryocoolers that are managed at the observatory level. The cooling power of the four cryocoolers can then be shared among the three instruments and the telescope. The dissipation at $4.5 \mathrm{~K}$ comes from three main sources within FIP: the HEMTs; the ADR, which provides continuous cooling at $50 \mathrm{mK}$; and the mechanisms. The design includes thermal straps made of pure annealed copper for all stages, sized for the load and distance required. These copper straps, along with the indium used for thermal connections, totals approximately $4 \mathrm{~kg}$ for FIP. The majority of this mass is for conducting the heat dissipated at $4.5 \mathrm{~K}$ to a central heat sink on the instrument structure that is, in turn, connected to a heat sink on the instrument mounting plate to which the $4.5 \mathrm{~K}$ cryocooler heat exchanger is also connected. This scheme leads to temperature gradients that are less than $5 \mathrm{mK}$ across the $4.5 \mathrm{~K}$ portions of the instrument. A five-stage CADR is planned for detector cooling (Fig. 7). Heat to the detectors is intercepted at two locations: an outer guard that includes a cooled filter window and the array itself, which also includes the microwave SQUID multiplexer (Fig. 8).

The five-stage CADR is currently being advanced from TRL 4 to TRL 6 through a grant from the Strategic Astrophysics Technology Program. This CADR will be capable of providing $6 \mu \mathrm{W}$ of cooling continuously at $50 \mathrm{mK}$, which is more than an order of magnitude better than the unit flown on Hitomi. ${ }^{25}$ Table 2 summarizes the calculated heat load and available lift with the cooler for each of the three FIP cooling stages. Heat loads at $50 \mathrm{mK}$ are roughly evenly divided between harnesses, a rigid strut suspension, and internal dissipation within the detectors and SQUID multiplexer. The first stage HEMT amplifiers are located at the $4.5 \mathrm{~K}$ stage. The first stage HEMT heat dissipation shown in the table corresponds to that of commercially available amplifiers operating at up to $8 \mathrm{GHz}$, with low noise and $10 \mathrm{~dB}$ of gain (at this relatively low gain the heat dissipation is being kept minimal). We are currently performing studies to verify this amplifier noise is as advertised. Parasitic conduction in harnesses is book-kept at the observatory level but is much smaller than the amplifier dissipation. 
Staguhn et al.: Far-infrared imager and polarimeter for the origins space telescope

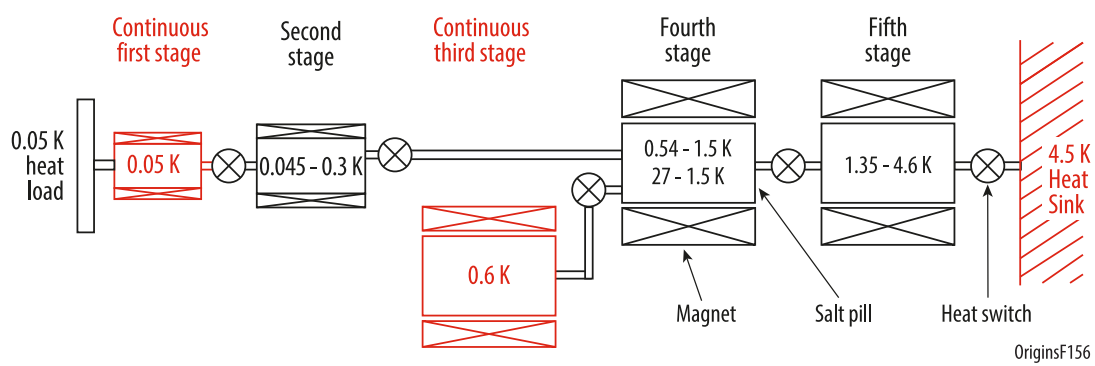

Fig. 7 The FIP CADR provides continuous cooling at two stages: $50 \mathrm{mK}$ (with $0.4 \mu \mathrm{K}$ stability) and $0.7 \mathrm{~K}$ (with $0.1 \mathrm{mK}$ stability), and deposits its heat at a $4.5 \mathrm{~K}$ heat sink.

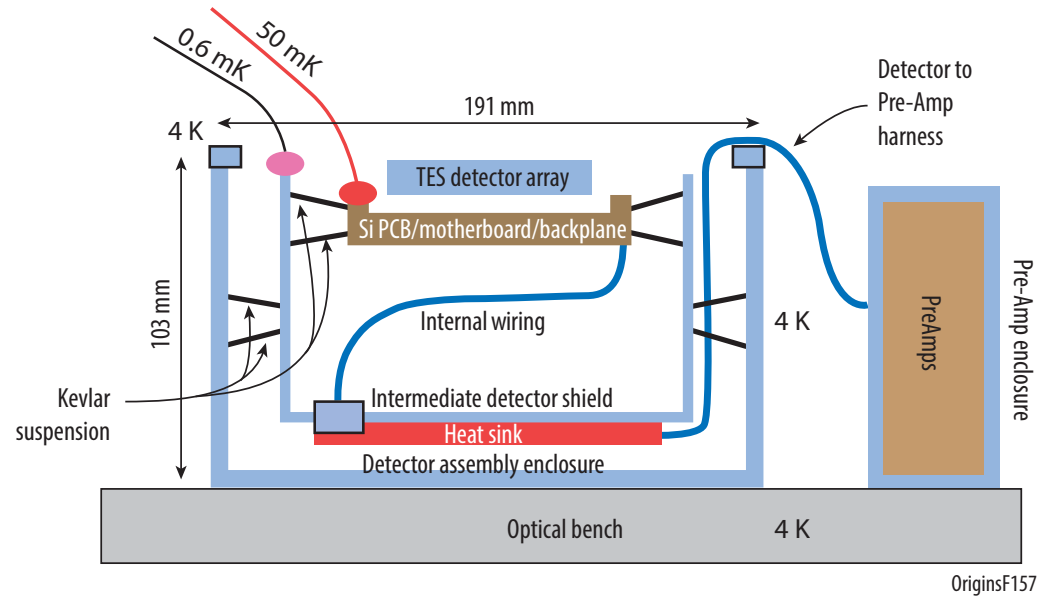

Fig. 8 The FIP focal plane array is cooled to $0.05 \mathrm{~K}$ and suspended within a $0.7 \mathrm{~K}$ enclosure, providing thermal shielding from the $4.5 \mathrm{~K}$ stage. The Kevlar suspension, which is common to most instruments with low temperature detectors, provides for excellent stiffness while having a very low heat conductivity.

Table 2 Calculations of the heat loads and available lift on the FIP cryogenic stages show margins of at least $200 \%$ over the calculated heat load maintained at each temperature stage.

\begin{tabular}{llcccc}
\hline \hline $\mathrm{T}(\mathrm{K})$ & Item & Heat load & Total & Capability $^{\mathrm{a}}$ & Margin (\%) $^{2}$ \\
\hline \multirow{2}{*}{$\mathbf{0 . 0 5}$} & Harnesses & $0.16 \mu \mathrm{W}$ & - & - & - \\
& Dissipation & $0.17 \mu \mathrm{W}$ & $0.56 \mu \mathrm{W}$ & $6 \mu \mathrm{W}$ & 971 \\
& Suspension & $0.23 \mu \mathrm{W}$ & - & - & - \\
& Harnesses & $7 \mu \mathrm{W}$ & - & - & 252 \\
$\mathbf{0 . 7}$ & Suspension & $76 \mu \mathrm{W}$ & $83 \mu \mathrm{W}$ & - & - \\
& HEMTs & $3.1 \mu \mathrm{W}$ & - & $64 \mathrm{~mW}$ & 533 \\
$\mathbf{4 . 5}$ & Mechanisms & $3 \mathrm{~mW}$ & $10.1 \mathrm{~mW}$ & - & - \\
\hline \hline
\end{tabular}

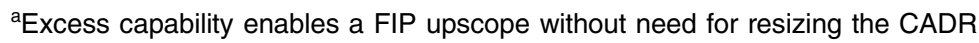

\section{Mechanical Architecture}

The FIP structure is shown in Fig. 4. Mechanical parts include exterior flexure mounts, a housing frame, thin enclosure, optical bench, and brackets that hold the three main mirrors. The design 
also includes mechanical components associated with the internal optics and detector assemblies. The material for all the mechanical components is beryllium for its light-weight and thermal conductivity properties.

\subsection{Mechanisms}

FIP has six mechanisms listed with their requirements and operational concepts in Table 3.

\subsection{FIP Instrument Control}

Figure 9 shows the electrical instrument control system block diagram. The main electronics box includes specialized control boards and a radiation hardened LEON3 CPU. System features include:

Table 3 FIP mechanisms.

\begin{tabular}{|c|c|c|}
\hline Mechanism & Requirement & Design concept \\
\hline HWP flip (2) & $\begin{array}{l}\text { Three positions, open center; element } \\
\text { diameter: } 150 \mathrm{~nm} \text { life: } 5 \text { y, } 22 \mathrm{k} \text { cycles + } \\
\text { test; duty cycle: low }\end{array}$ & $\begin{array}{l}\text { Superconducting stepper motor drives crank } \\
\text { between toggle positions, rotating element } \\
\text { housings around axis parallel to optical axis; } \\
\text { stop motor at center position to achieve open } \\
\text { condition }\end{array}$ \\
\hline $\begin{array}{l}\text { HWP } \\
\text { rotation (2) }\end{array}$ & $\begin{array}{l}\text { Rotate HWP at } 60 \mathrm{rpm} \text { read position to } \\
+/-0.1 \mathrm{deg} \text {; life: } 107 \text { revolutions; duty } \\
\text { cycle: high }\end{array}$ & $\begin{array}{l}\text { Superconducting brushless DC motor and } \\
\text { resolver to rotate HWP and sense its position }\end{array}$ \\
\hline Polarizer flip & $\begin{array}{l}\text { Two positions; element diameter: } 150 \mathrm{~mm} \text {; } \\
\text { life: } 5 \text { y, } 22 \mathrm{ft} \text { cycles + test; duty cycle: low }\end{array}$ & $\begin{array}{l}\text { Superconducting stepper motor drives crank } \\
\text { between toggle positions, rotating element } \\
\text { housings around axis parallel to optical axis }\end{array}$ \\
\hline Lens flip & $\begin{array}{l}\text { Two positions; element diameter: } 150 \mathrm{~mm} \text {; } \\
\text { elements move simultaneously; life: } 5 \mathrm{y} \text {, } \\
22 \text { k cycles + test; duty cycle: low }\end{array}$ & $\begin{array}{l}\text { Superconducting stepper motor drives crank } \\
\text { between toggle positions, translating linear } \\
\text { carriage }\end{array}$ \\
\hline
\end{tabular}

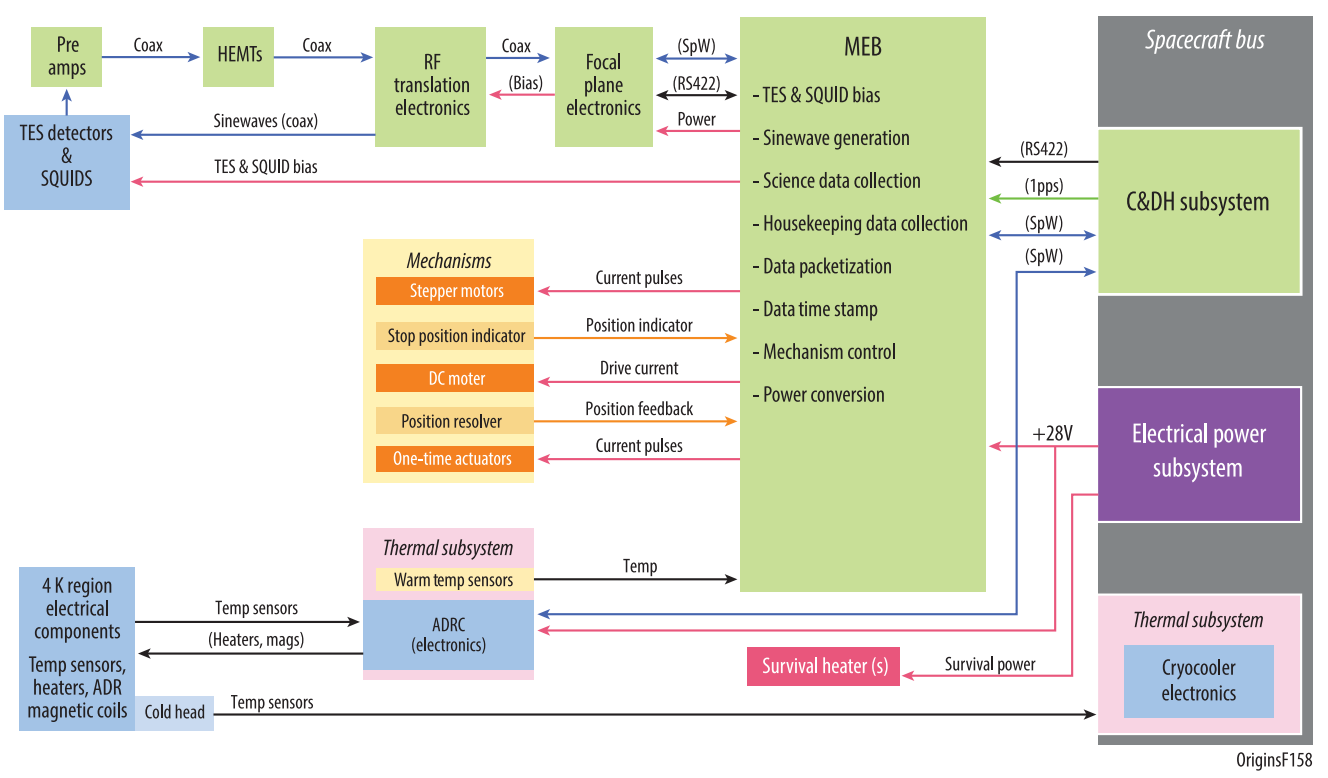

Fig. 9 This FIP block diagram shows how the instrument is controlled. 
- Mode management (standby, calibration, and execution of observing modes stare and mapping in total power and polarimetry modes)

- Instrument support (command processing, data collection, and support for firmware updates)

- Mechanism control

- Power distribution, including detector biasing

- Some degree of on-board autonomy (limit checking, some processing command sequences, and failure corrections)

\section{FIP Alignment, Integration, Test, and Calibration}

The FIP instrument will require its own unique facilities for testing and calibration at the instrument level before it is delivered for integration with the observatory. With its outer space background limited FIR detectors, the major requirement for the optical tests will be to provide an extreme dark environment for the tests with test sources (black body source and FIR laser) being attenuated to provide power levels suitable for the detectors, which will allow for gain and optical performance measurements.

Aside from the normal vibration, acoustic, and electromagnetic interference (EMI) environmental testing, the FIP instrument will need a thermal vacuum test chamber that can be cooled to liquid helium temperatures and is large enough to enclose the complete cold section of the instrument. The chamber needs to be FIR light-tight enough so that stray light within the detectors' sensitivity wavelengths is no greater than the infrared background that would be expected on-orbit. This test chamber should allow the introduction of FIR signals at the expected levels of the on-orbit target sources and the ability to verify the optical design of the instrument. The facility will need to allow connection between the cold instrument assembly in the test chamber and the warm readout electronics over flight-like cables.

The warm electronics for FIP will not require any particular test facilities other than the usual facilities used to conduct vibration, acoustic, EMI, and thermal vacuum tests. A spacecraft simulator will be required to test command and data transfers between FIP and the spacecraft electronics. It will be necessary to develop specialized test hardware, but although the scope of this development will be significant, it is well defined, with no new technology development required. FIP instrument I\&T is expected to take 12 months, assuming all the external test support hardware and software is ready in time by the start of testing.

Although the vast majority of the FIP instrument calibration and verification/validation activities will take place before instrument delivery for observatory integration, verification of its performance within the integrated observatory will be necessary to ensure that it still performs as expected in end-to-end observatory testing before launch.

\section{FIP Heritage and Maturity}

FIP is a mature instrument design except for the detectors. The design, in particular its optical layout, is similar to the SOFIA/HAWC+ instrument, ${ }^{26,27}$ which has operated routinely for science observations since December 2016. HAWC+ on SOFIA uses three of GSFC's $40 \times 32$ pixel TES-based BUG detector arrays, a potential design for FIP. With 8000 pixels, the required detector array is only roughly a factor of three larger than one of the two focal plane arrays in HAWC+. In light of the fact that the targeted array architectures are tileable, this factor of three in pixel count is achievable now. In terms of detector sensitivity on a single TES pixel level, the required sensitivity has been demonstrated in the Lab. ${ }^{28}$ The dynamic range requirement might force using two TES per pixel, which could either be read out in parallel or series, the latter has been demonstrated at GSFC. ${ }^{29}$

\section{FIP Predicted Performance}

\subsection{Photometric Survey Time}

In survey mapping or pointed modes, FIP quickly reaches the confusion limit in the long wavelength band (in $32 \mathrm{~ms}$ at $250 \mu \mathrm{m}$ ), see also Table 1, but allows integrations of unprecedented 


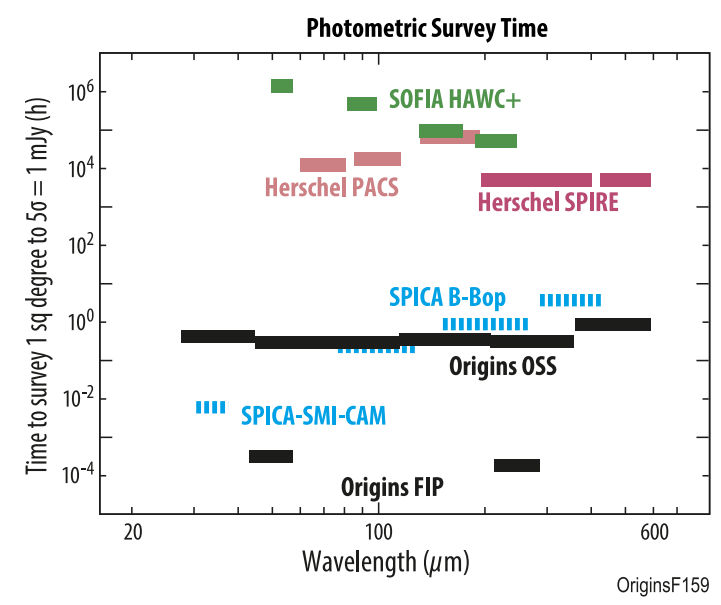

Fig. 10 A comparison of photometric survey times for a number of space-based FIR instruments. It is worthy to note that Origins/FIP will be the fastest mapping far-IR survey instrument ever.

depth and takes hours to reach the confusion limit in the $50 \mu \mathrm{m}$ band. With a detector NEP $=$ $3 \times 10^{-19} \mathrm{~W} / \sqrt{ } \mathrm{Hz}$, FIP will be dominated by astronomical backgrounds. ${ }^{1}$ The corresponding point source sensitivity is $0.9 \mu \mathrm{Jy}(5 \sigma, 1 \mathrm{~h})$ at $50 \mu \mathrm{m}, 2.5 \mu \mathrm{Jy}(5 \sigma, 1 \mathrm{~h})$ at $250 \mu \mathrm{m}$. It is worth noting that the confusion limit at $250 \mu \mathrm{m}$ does not allow an integration to $2.5 \mu \mathrm{Jy}$. The proposed surveys with FIP at this wavelength take the confusion limit into account. Additional performance parameters are provided in Table 1, whereas optical parameters for the telescope can be found in Ref. 30. Figure 10 shows the unprecedented mapping efficiency FIP will provide. Combined with the fast mapping capability of Origins, FIP can survey thousands of square degrees efficiently with significant advantages in continuum mapping speed over OSS, as shown in the same figure.

\section{References}

1. D. Farrah et al., "Review: far-infrared instrumentation and technological development for the next decade," J. Astron. Telesc. Instrum. Syst. 5(2), 020901 (2019).

2. H. Murakami et al., "The infrared astronomical mission AKARI," Publ. Astron. Soc. Jpn. 59(sp2), S369-S376 (2007).

3. M. F. Kessler, "The infrared space observatory (ISO)," Space Sci. Rev. 74, 57 (1995).

4. M. W. Werner et al., "The Spitzer space telescope mission," Astrophys. J. Suppl. Ser. 154, 1 (2004).

5.. G. L. Pilbratt et al., "Herschel Space Observatory. An ESA facility for far-infrared and submillimetre astronomy," Astron. Astrophys. 518, L 1 (2010).

6. T. L. Roellig et al., "Mid-infrared detector development for the Origins Space Telescope," J. Astron. Telesc. Instrum. Syst. 6, 041503 (2020).

7. C. M. Bradford et al., "The origins survey spectrometer (OSS): revealing the hearts of distant galaxies and forming planetary systems with far-IR spectroscopy," currently under review in JATIS.

8. A. Cooray et al., "Origins space telescope: from first light to life," Bull. Am. Astron. Soc. 51, 59 (2019).

9. P. A. Abell et al., "LSST science book, version 2.0," arXiv0912.0201L (2009).

10. D. Spergel et al., "WFIRST-2.4: what every astronomer should know," arXiv1305.5425S (2013).

11. M. Meixner et al., "Origins space telescope science to design traceability," currently under review in JATIS.

12. M. P. Viero et al., "HerMES: the contribution to the cosmic infrared background from galaxies selected by mass and redshift," Astrophys. J. 779, 32 (2013). 
13. D. P. Marrone et al., "Galaxy growth in a massive halo in the first billion years of cosmic history," Nature 553, 51 (2018).

14. R. Wang et al., "Star formation and gas kinematics of quasar host galaxies at $Z \sim 6$ : new insights from ALMA," Astrophys. J. 773, 44 (2013).

15. M. Negrello et al., "The detection of a population of submillimeter-bright, strongly lensed galaxies," Science 330, 800 (2010).

16. T. B. Miller et al., "A massive core for a cluster of galaxies at a redshift of 4.3," Nature 556, 469 (2018).

17. W. Fuhr et al., "Surface adjustment of the KOSMA 3m telescope using phase retrieval holography," Astron. Astrophys. 274, 975 (1993).

18. Zmuidzinas et al., "Overview paper on far-infrared detectors," currently under review in JATIS.

19. K. D. Irwin et al., "Microwave SQUID multiplexers for low-temperature detectors," Nucl. Instrum. Methods Phys. Res. 559, 802I (2006).

20. D. A. Bennett et al, "Microwave SQUID multiplexing for the Lynx x-ray microcalorimeter," J. Astron. Telesc. Instrum. Syst. 5(2), 021007 (2019).

21. J. Tuttle et al., "Development of a space-flight ADR providing continuous cooling at $50 \mathrm{mK}$ with heat rejection at $10 \mathrm{~K}$," IOP Conf. Ser. Mater. Sci. Eng. 278(1), 012009 (2017).

22. The Origins Mission Concept Study Team, "Origins technology development plan," 2019, https://asd.gsfc.nasa.gov/firs/docs/OriginsVolume2TechDevelopmentPlanREDACTED.pdf

23. H. T. Nguyen, "BICEP2/SPUD: searching for inflation with degree scale polarimetry from the South Pole," Proc. SPIE 7020, 70201F (2008).

24. C. Dragone, "Unique reflector arrangement with very wide field of view for multibeam antennas," Electron. Lett. 19(25), 1061 (1983).

25. T. Takahashi et al., "Hitomi (ASTRO-H) x-ray astronomy satellite," J. Astron. Telesc. Instrum. Syst. 4, 021402 (2018).

26. D. A. Harper et al., "HAWC +, the far-infrared camera and polarimeter for SOFIA," J. Astron. Instrum. 7, 1840008 (2018).

27. J. G. Staguhn et al., "Performance of backshort-under-grid kilopixel TES arrays for HAWC +," J. Low Temp. Phys. 184, 811 (2016).

28. T. Suzuki et al., "Development of ultra-low-noise TES bolometer arrays," J. Low Temp. Phys. 184, 52 (2016).

29. D. J. Benford et al., "Superconducting bolometer array architectures," Proc. SPIE 4855, 100 (2003).

30. J. A. Corsetti et al., "Optical design of the Origins Space Telescope," J. Astron. Telesc. Instrum. Syst. 7(1), 011010 (2021).

Johannes Staguhn is a principal research scientist at Johns Hopkins University. He received his PhD in physics from the University of Cologne, Germany. He joined NASA/GSFC in 2000, working on far-infrared instrumentation and astronomy. He is PI of the bolometer camera GISMO and deputy study scientist and instrument scientist for the Origins Space Telescope. Currently, he is leading the development of a mission concept for the characterization of the atmospheres of planets around M-stars.

Edward Amatucci has developed everything from robotic devices to scientific instruments for space missions over the past 38 years as an engineer, and currently is an instrument systems engineer as a contractor for NASA Goddard Space Flight Center. He received his Bachelor of Science in mechanical engineering from the University of Maryland, his Master of Engineering in mechanical engineering as well as a Master of Science in technical management from Johns Hopkins University.

Damon Bradley is head of the Instrument Electronics Development Branch and founder of the Digital Signal Processing Technology Group at NASA Goddard Space Flight Center, where he supervises the research and development work of more than 70 engineers and 30 projects. He is also co-investigator of 3 different spaceflight spectrometer systems and serves as an adjunct professor in the Electrical and Computer Engineering Department at UMBC. 
David Chuss is a professor of physics at Villanova University working in the area of astronomical instrumentation and specializing in polarimetry at wavelengths from the far-infrared through millimeter. He is interested in applying these techniques to a range of problems from understanding the role of magnetic fields in physical processes within the Milky Way to exploring the physics of the early universe through polarized measurements of the cosmic microwave background.

Mike DiPirro received his PhD in low-temperature physics from the State University of NY at Buffalo, and a one-year NRC Postdoctoral Fellowship at NIST. He joined NASA Goddard in 1980. He has worked on a number of astrophysics missions over the last 40 years including COBE, ASTRO-E, -E2, and -H, XRISM, Spitzer, WIRE, WISE, and JWST. Between COBE and ASTRO-E he was the PI on the Superfluid Helium On-Orbit Transfer Flight Demonstration, and Co-I on a Cross Enterprise Technology Development Program to develop a new type of adiabatic demagnetization refrigerator. Currently, he is the technical lead and chief technologist for the Origins Space Telescope study for the 2020 Astrophysics Decadal Survey.

Joseph Howard received his BS degree in physics from the US Naval Academy in Annapolis, Maryland, and his PhD in optical design from The Institute of Optics, University of Rochester, in Rochester, New York. He now serves as an optical designer for NASA, working on projects including the James Webb Space Telescope, the Roman Space Telescope, Origins, and the other future space missions.

David Leisawitz is NASA study scientist for the Origins Space Telescope and is most interested scientifically in the development of habitable conditions during planet formation. He was PI on the Space Infrared Interferometric Telescope mission concept study and served as NASA Goddard study lead for the Submillimeter Probe of the Evolution of Cosmic Structure. He was Mission Scientist for the Wide-field Infrared Survey Explorer and Deputy Project Scientist for the Cosmic Background Explorer.

Margaret Meixner is director of the SOFIA Science Mission Operations Center. She received bachelor's degrees in electrical engineering and math at University of Maryland, College Park, in 1987 and her PhD in astronomy at University of California, Berkeley, in 1993. Her research includes infrared instrumentation, and dust evolution in galaxies. She is a member of the JWST/MIRI Science Team, Community co-chair of NASA's STDT for the Origins Space Telescope mission concept, and formerly JWST project scientist and Distinguished Astronomer at STScI. She is a Fellow of the AAAS.

Alexandra Pope received her PhD from University of British Columbia. She is an associate professor of Astronomy at the University of Massachusetts Amherst. She is an observational astronomer who specializes in infrared, (sub)millimeter and radio observations of dusty galaxies at high redshift.

Joaquin Vieira is an associate professor of astronomy and physics at the University of Illinois at Urbana-Champaign. He received his $\mathrm{PhD}$ in physics in 2009 from the University of Chicago. He joined the faculty at University of Illinois in 2013. He is the PI of the Terahertz Intensity Mapper (TIM), a NASA-funded balloon experiment slated for an Antarctic flight in 2025.

Edward Wollack is a research astrophysicist in the Observational Cosmology Laboratory at NASA's Goddard Space Flight Center. His interests include cosmology, astronomical instrumentation, and electromagnetics.

Biographies of the other authors are not available. 